\title{
How Can We Improve the Quality of Scientific Research and Publications? Guidelines for Authors, Editors, and Reviewers
}

Scientific research (observational, clinical, and metaanalyses/systematic reviews) applies scientific method to solve medical problems. When the studies are ethically and correctly planned, conducted, and reported, the results guide clinicians and medical decision-makers appropriately. Methodological weaknesses of scientific research may lead to invalid conclusions and a waste of resources. Today's evidence-based medicine requires high-level clinical trials with certain criteria. These criteria cover all four steps of scientific research, namely, study design, data collection, statistical analysis, and publication. Shortcomings in any of these steps may result in a low-quality paper that has little chance of being published.

In the vast area of methodology- and publication-related issues, there are two important tools to help researchers improve their studies. These are: 1. guidelines, and 2. clinical trial registration systems.

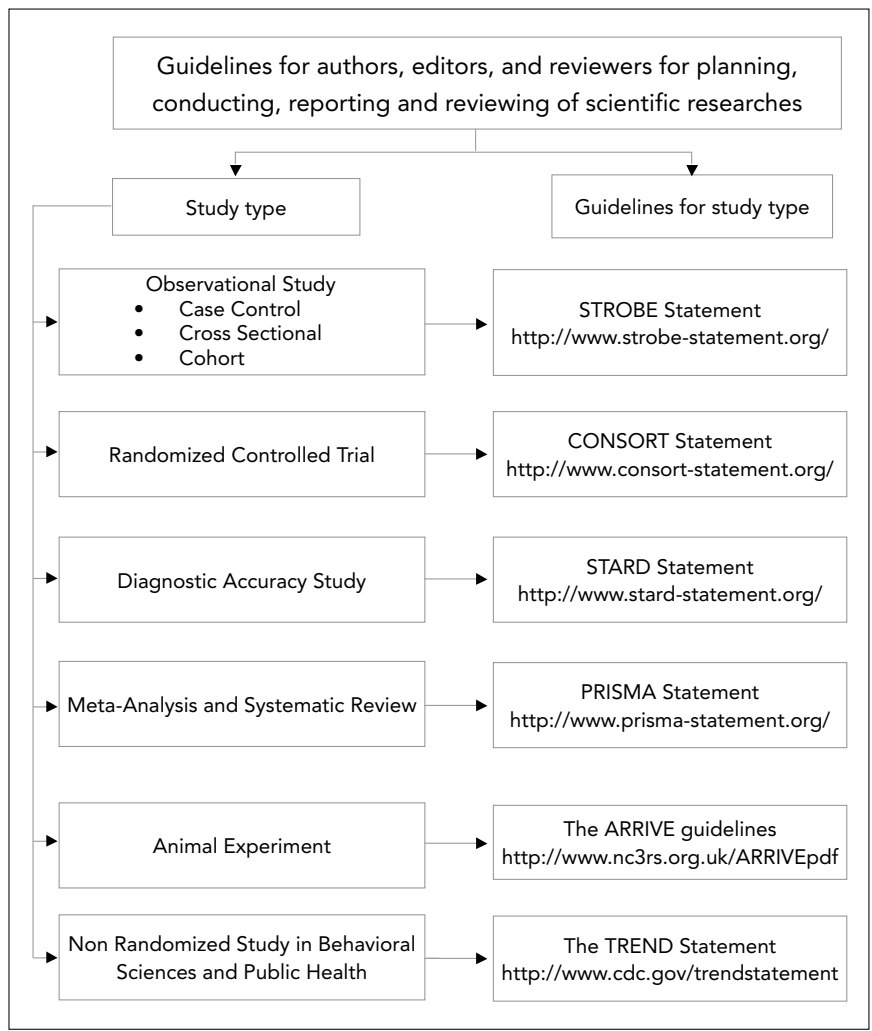

Figure 1. Flowchart of the selection of guidelines by study type

\section{Guidelines}

Guidelines set pre-determined criteria for studies in a checklist format. These criteria cover planning, conducting, and publication stages. The guidelines also help journal editors and referees in their review efforts (1).

Many respected medical journals, as well as the Council of Science Editors, the International Committee of Medical Journal Editors (ICMJE), and the World Association of Medical Editors (WAME) support the use of these guidelines. Despite their advantages, the use of guidelines is not at the desired level. In a recent paper, Marusic et al. assessed the endorsement of reporting guidelines in rheumatology journals, and found a low endorsement rate (2).

Various guidelines have been developed for different types of studies. The CONSORT (CONsolidated Standards Of Reporting Trials) and STROBE (STrengthening the Reporting of OBservational studies in Epidemiology) statements are examples of the most commonly endorsed and used types of guidelines. Figure 1 presents a flowchart that shows the selection of appropriate guidelines according to the study type. For further information on the reporting guidelines for health research, researchers and authors may also refer to the website of the EQUATOR (Enhancing the QUAlity and Transparency Of health Research) network (www.equator-network. org/), an international initiative for improving the quality of research and publications. A detailed catalogue of reporting guidelines for health research is also available on the same website (www.equator-network.org/index.aspx?o=4598).

\section{The Clinical Trial Registration System}

In this system, protocols of clinical trials carried out on humans all over the world can be defined and obtained. This system aims to help ensure transparency in clinical trials. The ICMJE recommends that clinical trials are registered before their results are reported.

Clinical trials can be registered at the http://clinicaltrials. gov/ website or at one of the registries of the WHO's International Clinical Trials Registry Platform (www.who.int/ictrp/ network/primary/en/index.html). A clinical trial registration number beginning with the abbreviation of the registry followed by a number is obtained (e.g., NCT00000000). Ongoing and completed clinical trial protocols can be accessed on the registry websites. Reports of clinical trials that have been registered at these data providers may have a higher chance of being accepted for publication. 
In conclusion, the Balkan Medical Journal strongly recommends that researchers/authors obtain a clinical trial registration number for their prospective clinical trials. Moreover, the establishment of national clinical trial registration system should be considered. In addition, we encourage authors, editors, and referees to use the appropriate guideline to improve the quality of their scientific research and the resulting publications.

\section{Associate Professor Necdet SÜT, PhD \\ Biostatistics Editor, Balkan Medical Journal \\ Department of Biostatistics and Medical Informatics, \\ Trakya University Faculty of Medicine, Edirne, Turkey}

\section{References}

1. Süt $N$, Senocak $M$, Uysal $O$, Köksalan $H$. Assessing the quality of randomized controlled trials from two leading cancer journals using the CONSORT statement. Hematol Oncol Stem Cell Ther 2008;1:38-43.

2. Marusic A, Gasparyan AY, Kitas GD. Promoting transparent and accurate reporting of research studies in rheumatology:Endorsement of reporting guidelines in rheumatology journals, Semin Arthritis Rheum 2013 doi:10.1016/j.semarthrit.2013.01.005. [Epub ahead of print]. [CrossRef] 